\title{
MODULES WHOSE CERTAIN SUBMODULES ARE ESSENTIALLY EMBEDDED IN DIRECT SUMMANDS
}

\author{
YELIZ KARA AND ADNAN TERCAN
}

\begin{abstract}
It is well known that, if the ring has acc on essential right ideals, then for every quasi-continuous module over the ring, the finite exchange property implies the full exchange property. In this paper, we obtain the former implication for the generalizations of quasi-continuous modules over a ring with acc on right annhilators of elements of the module. Moreover, we focus on direct sums and direct summands of weak $C_{12}$ modules i.e., modules with the property that every semisimple submodule can be essentially embedded in a direct summand. To this end, we prove that since weak $C_{12}$ is closed under direct sums. Amongst other results, we provide several counterexamples including the tangent bundle of a real sphere of odd dimension over its coordinate ring for the open problem of whether weak $C_{12}$ implies the $C_{12}$ condition.
\end{abstract}

1. Introduction. All rings are associative with unity and modules are unital right modules. We use $R$ to denote such a ring and $M$ to denote a right $R$-module. Recall that a module is called extending or $C S$ or said to satisfy the $C_{1}$ condition if every submodule is essential in a direct summand; equivalently, every complement submodule is a direct summand. This condition has proved to be an important common generalization of the injective, semisimple and uniform module notions. There have been a number of generalizations of the extending property, including the following:

(1) $M$ is a weak $C S$ [10] if every semisimple submodule of $M$ is essential in a direct summand of $M$;

(2) $M$ is a $C_{11}$-module [12] if each submodule of $M$ has a complement that is a direct summand of $M$;

2010 AMS Mathematics subject classification. Primary 16D70, Secondary 16D50, 16D80.

Keywords and phrases. Extending module, exchange property, $C_{12}$-module, tangent bundle.

Received by the editors on January 14, 2014, and in revised form on June 3, 2014. 
(3) $M$ is a $C_{12}$-module [12] if each submodule can be essentially embedded in a direct summand of $M$, and

(4) $M$ is $P I$-extending [3] if every projection invariant submodule (i.e., every submodule such that the image under all idempotent endomorphisms contained in itself) is essential in a direct summand of $M$.

In a similar way to weak $C S$-modules [10], weak $C_{11}$ and weak $C_{12}$ modules were introduced in $[\mathbf{6 , 1 5}$. Recall that a module $M$ is a weak $C_{11}\left(C_{12}\right)$-module if each semisimple submodule of $M$ has a complement that is a direct summand (if each semisimple submodule of $M$ can be essentially embedded in a direct summand) of $M$.

In this paper, we prove that if the ring $R$ has acc (ascending chain condition) on right annihilators $r(m)=\{r \in R \mid m r=0\}$, where $m \in M$ and $M_{R}$ satisfy $C_{11}$ and $C_{3}$ (or $C_{12}$ and $C_{2}$ ) then the finite exchange property implies the full exchange property. We also obtain the $P I$-extending version of the result on continuous modules [9, Proposition 3.5] when the endomorphism ring of the module is Abelian.

Further, we focus our attention on weak $C_{12}$-modules as a proper generalization of extending modules. It is well known that a direct summand of an extending module is extending, but a direct sum of extending modules is not an extending module, e.g., let $M$ be the $\mathbb{Z}$ module $(\mathbb{Z} / \mathbb{Z} p) \oplus\left(\mathbb{Z} / \mathbb{Z} p^{3}\right)$, where $p$ is any prime integer (see [12, page 1814]). In contrast to extending modules, we show that any direct sum of weak $C_{12}$-modules is a weak $C_{12}$-module. Since we are unable to settle at this time whether a direct summand of a weak $C_{12}$-module needs to be a weak $C_{12}$-module we obtain a positive answer for this question under some conditions. Recall that, whether weak $C_{12}$ implies $C_{12}$ was left as a problem in [6, page 496]. However, we provide several counterexamples which exhibit the failure of the problem. To this end, we observe that tangent bundles of all real spheres of odd dimensions over their coordinate rings have weak $C_{12}$ but not the $C_{12}$ property. We have then, for any module, the following implications:

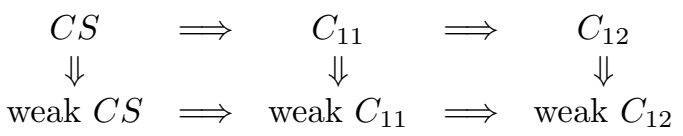


No other implications can be added to this table in general. To see why this is the case, we refer to [12, page 1814], [12, Proposition 3.6], [10, Example 1.1], Example 3.7, [17, Counter Example 3] and [13, Proposition 2.6].

Let $R$ be any ring and $M$ a right $R$-module. If $X \subseteq M$, then $X \leq M$ and Soc $X$ denote $X$ as a submodule of $M$ and the socle of $X$, respectively. A module $M$ is called locally Noetherian if every finitely generated submodule is Noetherian. Further, a ring is called Abelian if every idempotent is central. For any unexplained terminology, definitions and notation, see $[\mathbf{1}, \mathbf{4}, \mathbf{5}, \mathbf{7}, \mathbf{9}]$.

2. Generalizations of extending modules with $C_{2}$ or $C_{3}$. Let $R$ be a ring and $M$ a right $R$-module. Recall the following conditions for $M$ :

$C_{3}$ : for all direct summands $K$ and $L$ of $M$ with $K \cap L=0$, the submodule $K \oplus L$ is also a direct summand of $M$.

$C_{2}$ : for each direct summand $N$ of $M$ and each monomorphism $\varphi: N \rightarrow M$, the submodule $\varphi(N)$ is also a direct summand of $M$.

A $C S$-module with $\left(C_{3}\right) C_{2}$ is called a (quasi) continuous module. For good references on these notions, see $[\mathbf{5}, \mathbf{9}]$. In this section, we mainly work with the general form of (quasi) continuous modules. We begin by proving a basic fact about indecomposable modules with Goldie dimension 1.

Lemma 2.1. Let $R$ be a right Noetherian ring and $M$ a nonzero indecomposable right $R$-module. Then the following conditions are equivalent:

(i) $M$ is uniform,

(ii) $M$ has $C_{11}$,

(iii) $M$ has $C_{12}$,

(iv) $M$ is $P I$-extending.

Proof.

(i) $\Rightarrow$ (ii) is clear.

(ii) $\Rightarrow$ (iii). By [12, Proposition, 3.2] 
(iii) $\Rightarrow$ (iv). By hypothesis, every submodule of $M$ is projection invariant in $M$. Let $X$ be a nonzero submodule of $M$. Then there exists a nonzero element $x \in X$ and $R / r(x) \cong x R$. Thus, there exists a uniform submodule $U$ of $M$ such that $U \leq x R \leq X \leq M$. By hypothesis, there exists a monomorphism $\varphi: \bar{U} \rightarrow M$ such that $\varphi(U)$ is essential in $M$. Since $\varphi(U) \leq_{e} M$ and $\varphi$ is a monomorphism, we have $U$ is essential in $M$, and hence $X$ is essential in $M$.

(iv) $\Rightarrow$ (i). Let $N$ be a nonzero submodule of $M$. Since $M$ is indecomposable, $N$ is a projection invariant submodule of $M$. By (iv), there exists a direct summand $K$ of $M$ such that $N$ is essential in $K$. Hence, $K=M$. Thus, $N$ is essential in $M$ so $M$ is uniform.

Corollary 2.2. Let $R$ be a right locally Noetherian ring and $M a$ nonzero indecomposable right $R$-module. Then the following statements are equivalent.

(i) $M$ is uniform,

(ii) $M$ has $C_{11}$,

(iii) $M$ has $C_{12}$,

(iv) $M$ is $P I$-extending.

Proof. The proof is immediate by Lemma 2.1 .

Lemma 2.3. Let $R$ be a ring and $M$ an $R$-module such that $R$ satisfies acc on right ideals of the form $r(m)$ where $m \in M$. If $M_{R}$ satisfies

(a) $C_{11}$ and $C_{3}$,

or

(b) $C_{12}$ and $C_{2}$,

then $M$ has an indecomposable decomposition.

Proof. The proof follows from [12, Lemma 4.6] and [9, Theorem 2.17].

Proposition 2.4. The following statements are equivalent for a nonsingular $C_{11}$-module $M$ which satisfies $C_{3}$.

(i) $M$ has an indecomposable decomposition. 
(ii) Every finitely generated submodule of $M$ has finite uniform dimension.

(iii) Every cyclic submodule of $M$ has finite uniform dimension.

(iv) $R$ satisfies acc on right ideals of the form $r(m)$ where $m \in M$.

Proof. (i) $\Rightarrow$ (ii). There exist an index set $I$ and indecomposable submodules $M_{i}(i \in I)$ of $M$ such that $M=\oplus_{i \in I} M_{i}$. Since $M$ is $C_{11}$-module with $C_{3}, M_{i}$ is also $C_{11}$ by [12, Theorem 4.3]. It follows that $M_{i}(i \in I)$ is uniform. If $L$ is a finitely generated submodule of $M$, then $L \subseteq \oplus_{i \in J} M_{i}$ for some finite subset $J$ of $I$, and hence, $L$ has finite uniform dimension.

(ii) $\Rightarrow$ (iii). Clear.

(iii) $\Rightarrow$ (iv). Let $m \in M$. Suppose that $r(m)$ is essential in a right ideal $A$ of $R$. Let $a \in A$. There exists an essential right ideal $E$ of $R$ such that $a E \subseteq r(m)$. It follows that $m a E=0$, and hence, $m a=0$ so $a \in r(m)$. Thus, $r(m)=A$. Hence, $r(m)$ is a complement in $R$-module $R$ for each $m \in M$. Moreover, $R / r(m) \cong m R$, gives that the $R$-module $R / r(m)$ has finite uniform dimension. Now (iv) follows by [5, Theorem 5.10].

(iv) $\Rightarrow$ (i). Follows by $[\mathbf{1 2}$, Theorem 4.7] and [9, Theorem 2.17].

Combining Proposition 2.4 together with [18, Corollary 6] gives the following result on the exchange property of modules without further proof.

Theorem 2.5. Let $M$ be a nonsingular module such that $m R$ has finite uniform dimension for each $m \in M$. If $M$ satisfies $C_{11}$ and $C_{3}$, then the finite exchange property implies the full exchange property.

Corollary 2.6. Let $R$ be a ring with finite uniform dimension and $M$ a nonsingular right $R$-module which satisfies $C_{11}$ and $C_{3}$. Then the finite exchange property implies the full exchange property.

Proof. Let $M$ and $R$ be as stated. Let $m \in M$. Then $r(m)$ is a complement in the right $R$-module $R$. Hence, the $R$-module $R / r(m)$ has finite uniform dimension. It is clear that $m R \cong R / r(m)$. Now apply Theorem 2.5 to get the result. 
Recall that, over a ring with acc on essential right ideals, the finite exchange property implies the full exchange property for every quasicontinuous module [16]. We have the following result in this trend.

Theorem 2.7. Let $R$ be a ring with acc on right ideals of the form $r(m)$ where $m \in M$ and $M$ is a right $R$-module. If $M_{R}$ satisfies

(a) $C_{11}$ and $C_{3}$,

or

(b) $C_{12}$ and $C_{2}$,

then the finite exchange property implies the full exchange property.

Proof. By Lemma 2.3, $M$ has an indecomposable decomposition. Then the result follows from [18, Corollary 6].

The next example shows that the assumptions of the above theorem do not imply the quasi-continuity of the module.

Example 2.8. Let $M$ denote the $\mathbb{Z}$-module $\left(\mathbb{Z} / \mathbb{Z}_{p}\right) \oplus \mathbb{Q}$ for any prime $p$. Then $M$ satisfies $C_{11}$ and $C_{2}$ as well as $C_{12}$ and $C_{3}$ by [12, Proposition 3.2] and [9, Proposition 2.2]. Since $M_{\mathbb{Z}}$ is not a $C S$ module, it is not a quasi-continuous module (see [12, Example 4.2]).

Corollary 2.9. Let $M$ be a locally Noetherian module with $C_{11}$ and $C_{3}$. Then the finite exchange property implies the full exchange property.

Proof. Let $m \in M$. Since $R / r(m) \cong m R, R / r(m)$ is a Noetherian right $R$-module. It follows that $R$ satisfies acc on right ideals of the form $r(x), x \in M$. Then Theorem 2.7 yields the result.

One might ask whether a $P I$-extending module with the full exchange property and $C_{3}$ condition (or $C_{2}$ ) implies that the endomorphism ring of the module is Abelian or not? However, we provide examples which eliminate these possibilities.

Example 2.10. Let $R=\mathbb{Z}_{(p)}$ be the localization of integers $\mathbb{Z}$ at a prime $p$. Put $M_{R}=\mathbb{Z}_{(p)} \oplus \mathbb{Z}\left(p^{\infty}\right)$. Then $M_{R}$ is a $P I$-extending module with $C_{3}$ which does not satisfy $C_{2}$. Moreover, $M$ has the full exchange property whose endomorphism ring has noncentral idempotents. 
Proof. Observe that $M_{R}$ is quasi-continuous by [16, Example 2.13]. Hence, $M_{R}$ is $P I$-extending with $C_{3}$ by [3, Proposition 3.7]. Now, let us consider $f \in \operatorname{End}\left(M_{R}\right)$ such that $f(x, y)=(p x, y)$ where $(x, y) \in M$. It is easy to see that $f$ is a monomorphism and $f(M)=p \mathbb{Z}_{(p)} \oplus \mathbb{Z}\left(p^{\infty}\right)$ which is an essential submodule of $M$. By [9, Lemma 3.14], $M_{R}$ does not satisfy the $C_{2}$ condition. For the last part, see [16, Example 2.13].

Example 2.11. Let $M_{2}(R)$ be the matrix ring as in [2, Example 2.7]. Let $T=M_{2}(R)$. Since $T_{T}$ is a $C_{11}$-module then $T_{T}$ is a $P I$-extending module by [3, Proposition 3.7]. Note that $T_{T}$ has the full exchange property by [9, Theorem 3.24]. Moreover, it can be seen easily that the endomorphism ring of $T$ is not Abelian.

The next few results concern the endomorphism ring of $P I$-extending modules. To this end, we refer to $[\mathbf{1 4}, \mathbf{1 5}]$ for the corresponding results in terms of $C_{11}$-modules and weak $C_{11}$-modules, respectively. We will use $S$ and $J(S)$ to denote the endomorphism ring of a module $M$ and the Jacobson radical of $S$, respectively. Further, $\Delta$ will stand for the ideal $\{\alpha \in S \mid \operatorname{ker} \alpha$ is essential in $M\}$.

Theorem 2.12. Let $M_{R}$ be a PI-extending module with the $C_{2}$ condition, and let $S$ be an Abelian ring. Then $S / \Delta$ is a (von Neumann) regular ring and $\Delta=J(S)$.

Proof. Let $\alpha \in S, K=\operatorname{ker} \alpha$. Let $f^{2}=f \in S$, and let $y \in$ $f(K)$. Then there exists an element $k$ of $K$ such that $y=f(k)$. So $\alpha(y)=\alpha(f(k))$. Therefore, $\alpha(y)=\alpha(f(k))=f(\alpha(k))=0$. Hence, $y \in K$. It follows that $K$ is a projection invariant submodule of $M$. By hypothesis, there exists a direct summand $L$ of $M$ such that $L$ is a complement of $K$ in $M$. Since $\left.\alpha\right|_{L}$ is a monomorphism then $\alpha(L)$ is a direct summand of $M$, by $C_{2}$. Hence, there exists $\beta \in S$ such that $\beta \alpha=\left.1\right|_{L}$. Then

$$
(\alpha-\alpha \beta \alpha)(K \oplus L)=(\alpha-\alpha \beta \alpha)(L)=0,
$$

and so $K \oplus L$ is a submodule of $\operatorname{ker}(\alpha-\alpha \beta \alpha)$. Since $K \oplus L$ is essential in $M$ then $\alpha-\alpha \beta \alpha \in \Delta$. Therefore, $S / \Delta$ is a regular ring. This also proves that $J$ is contained in $\Delta$. 
Let $a \in \Delta$. Since ker $a \cap \operatorname{ker}(1-a)=0$ and $\operatorname{ker} a$ is essential in $M$ then $\operatorname{ker}(1-a)=0$. Hence, $(1-a) M$ is a direct summand of $M$ by $C_{2}$. However, $(1-a) M$ is essential submodule of $M$ since ker $a$ is a submodule of $(1-a) M$. Thus, $(1-a) M=M$, therefore $1-a$ is a unit in $S$. Hence, $a \in J$. It follows that $\Delta=J$.

Corollary 2.13. Let $M$ be a nonsingular right $R$-module. If $M$ is a $P I$-extending module with $C_{2}$ condition and $S$ is Abelian, then $S$ is a regular ring.

Proof. Let $g \in \Delta$ and $N=\operatorname{ker} g$. Then, for any $x \in M$, we build up the following set

$$
L=\{r \in R \mid x r \in N\} .
$$

Then clearly $L$ is a right ideal of $R$ and also $L$ is essential in $R$. Now, $g(x) L=0$. Since $M$ is nonsingular then $g(x)=0$, and since $x$ is arbitrary, $g=0$. Therefore, $\Delta=0$. Hence, the result follows from Theorem 2.8.

Note that $C_{2}$ cannot be replaced by $C_{3}$ in Theorem 2.12 as the following example illustrates. Let $M$ denote the $\mathbb{Z}$-module $\mathbb{Z}$. Obviously, $M_{\mathbb{Z}}$ is a $P I$-extending module with $C_{3}$ and $S=\operatorname{End}\left(M_{\mathbb{Z}}\right) \cong \mathbb{Z}$ is Abelian. But $\Delta=\operatorname{End}\left(M_{\mathbb{Z}}\right) \cong \mathbb{Z}$ and $0=J(S) \neq \Delta$.

We conclude this section with the following example which demonstrates both Theorem 2.7 and Theorem 2.12 .

Example 2.14. Let $M$ be the module as in Example 2.8, i.e., let $M$ be the $\mathbb{Z}$-module $\left(\mathbb{Z} / \mathbb{Z}_{p}\right) \oplus \mathbb{Q}$. Then $M_{\mathbb{Z}}$ is a $P I$-extending module with $C_{2}$ and the endomorphism ring of $M$ is Abelian.

Proof. From Example 2.8 and [3, Proposition 3.7], $M_{\mathbb{Z}}$ is a PIextending module. Moreover, [12, Example 4.2] shows that $M_{\mathbb{Z}}$ satisfies the $C_{2}$ property. Now,

$$
S=\operatorname{End}(M) \cong\left[\begin{array}{cc}
\operatorname{End}(\mathbb{Z} / \mathbb{Z} p) & \operatorname{Hom}(\mathbb{Q}, \mathbb{Z} / \mathbb{Z} p) \\
\operatorname{Hom}(\mathbb{Z} / \mathbb{Z} p, \mathbb{Q}) & \operatorname{End}(\mathbb{Q})
\end{array}\right]=\left[\begin{array}{cc}
\mathbb{Z} / \mathbb{Z} p & 0 \\
0 & \mathbb{Q}
\end{array}\right]
$$

which is Abelian. 
3. Weak $C_{12}$ modules. In this section we focus our attention on weak $C_{12}$ modules, i.e., modules with the property that each semisimple submodule can be essentially embedded in a direct summand. Recall that a direct sum of extending modules is not an extending module in general (see, for example, Example 2.14). However, we have the following closure property which shows that any direct sum of weak $C_{12}$ modules are also a weak $C_{12}$-module.

Theorem 3.1. Any direct sum of modules with the weak $C_{12}$ property satisfies weak $C_{12}$.

Proof. Let $M_{\lambda}(\lambda \in \Lambda)$ be a nonempty collection of modules, each satisfying weak $C_{12}$. Let $M=\oplus_{\lambda \in \Lambda} M_{\lambda}$, and let $N$ be any semisimple submodule of $M$. Let $\Lambda^{\prime}$ be a nonempty subset of $\Lambda$ containing $\lambda$ such that $M^{\prime}=\oplus_{\lambda \in \Lambda^{\prime}} M_{\lambda}$. Assume that $M^{\prime}$ satisfies the weak $C_{12}$ property. Then for any semisimple submodule $N^{\prime}$ of $M^{\prime}$ there exists a direct summand $K^{\prime}$ of $M^{\prime}$ and a monomorphism $\alpha_{1}: N^{\prime} \rightarrow K^{\prime}$ such that $\alpha_{1}\left(N^{\prime}\right)$ is essential in $K^{\prime}$. Suppose $\Lambda \neq \Lambda^{\prime}$. Then there exists $\mu \in \Lambda$, $\mu \notin \Lambda^{\prime}$. Let $\Lambda^{\prime \prime}=\Lambda^{\prime} \cup\{\mu\}$ and $M^{\prime \prime}=\oplus_{\lambda \in \Lambda^{\prime \prime}} M_{\lambda}=M^{\prime} \oplus M_{\mu}$. Since $M_{\mu}$ is a weak $C_{12}$ module, for any semisimple submodule $N_{\mu}$ of $M_{\mu}$ there exist a direct summand $K_{\mu}$ of $M_{\mu}$ and a monomorphism $\alpha_{2}: N_{\mu} \rightarrow K_{\mu}$ such that $\alpha_{2}\left(N_{\mu}\right)$ is essential in $K_{\mu}$. It is clear that $K^{\prime} \cap K_{\mu}=0$. Let $K^{\prime \prime}=K^{\prime} \oplus K_{\mu}$. Note that $K^{\prime \prime}$ is a direct summand of $M^{\prime \prime}$. Consider the semisimple submodule $N^{\prime} \oplus N_{\mu}$ of $M^{\prime \prime}$. Define $\beta: N^{\prime} \oplus N_{\mu} \rightarrow K^{\prime} \oplus K_{\mu}$ by

$$
\beta(n)=\beta\left(m_{1}+m_{2}\right)=\alpha_{1}\left(m_{1}\right)+\alpha_{2}\left(m_{2}\right),
$$

where $n \in N^{\prime} \oplus N_{\mu}, m_{1} \in N^{\prime}$ and $m_{2} \in N_{\mu}$. It is easy to check that $\beta$ is a monomorphism. Furthermore,

$$
\beta\left(N^{\prime} \oplus N_{\mu}\right)=\alpha_{1}\left(N^{\prime}\right) \oplus \alpha_{2}\left(N_{\mu}\right)
$$

is an essential submodule of $K^{\prime} \oplus K_{\mu}$ by [1, Proposition 5.20 ]. When using the transfinite induction argument, there exist a direct summand $K$ of $M$ and a monomorphism $\gamma: N \rightarrow K$ such that $\gamma(N)$ is essential in $K$. Thus, $M$ satisfies weak $C_{12}$.

The proof of the above theorem brings us to the following observation. Although the statement of [13, Theorem 1.2] is true, its proof is not complete at all. Incidentally, the idea in the proof of Theorem 3.1 
works exactly by replacing semisimple submodules with submodules to obtain [13, Theorem 1.2].

Corollary 3.2. Any direct sum of modules which satisfy the weak $C_{11}$ (respectively, one of the conditions, extending, uniform or $C_{11}$ ) satisfy weak $C_{12}$.

Proof. Immediate by Theorem 3.1 .

After applying Theorem 3.1, we have the next easy fact on modules over Dedekind domains.

Corollary 3.3. Let $R$ be a Dedekind domain and $M$ an $R$-module with finite uniform dimension. Then $M$ is a weak $C_{12}$ module.

Proof. It follows from [10, Corollary 1.17] and Corollary 3.2.

Recall that $C_{11}$ and also $C_{12}$ properties are not inherited by direct summands (for details see, $[\mathbf{1 1}, \mathbf{1 3}, \mathbf{1 5}]$ ). We do not know whether direct summands of a weak $C_{12}$-module need to be weak $C_{12}$ or not, so far. Now we deal with some special cases for the former question.

Corollary 3.4. Let $M$ be a right $R$-module and $M=U \oplus V$ where $U$ and $V$ are uniform submodules. Then every direct summand of $M$ is a weak $C_{12}$-module.

Proof. Let $0 \neq K$ be a direct summand of $M$. If $K=M$, then $K$ has a weak $C_{12}$ from Corollary 3.2. If $K \neq M$, then $K$ is uniform. Hence, $K$ has a weak $C_{12}$.

Theorem 3.5. Let $M$ be a $\mathbb{Z}$-module such that $M$ is a direct sum of uniform modules. Then every direct summand of $M$ is a weak $C_{12}$ module.

Proof. Let $N$ be a direct summand of $M$. Then $N$ is also a direct sum of uniform modules by [12, Theorem 5.5]. Now, Corollary 3.2 yields that $N$ satisfies weak $C_{12}$. 
The next result provides a condition which ensures that a direct summand of a module is a weak $C_{12}$-module.

Theorem 3.6. Let $M=M_{1} \oplus M_{2}$. Then $M_{1}$ satisfies weak $C_{12}$ if and only if, for every semisimple submodule $N$ of $M_{1}$, there exist a direct summand $K$ of $M$ and $\varphi$ monomorphism on $N$ such that $M_{2} \subseteq K$, $\varphi(N) \cap K=0$ and $\varphi(N) \oplus K$ is an essential submodule of $M$.

Proof. Suppose $M_{1}$ satisfies weak $C_{12}$. Let $N$ be any semisimple submodule of $M_{1}$. There exist a direct summand $L$ of $M_{1}$ and a monomorphism $\varphi: N \rightarrow L$ such that $\varphi(N)$ is an essential submodule of $L$. So $M_{1}=L \oplus L^{\prime}$ for some submodule $L^{\prime}$ of $M_{1}$. Clearly, $L^{\prime} \oplus M_{2}$ is a direct summand of $M,\left(L^{\prime} \oplus M_{2}\right) \cap \varphi(N)=0$ and $\left(L^{\prime} \oplus M_{2}\right) \oplus \varphi(N)$ is an essential submodule of $M$.

Conversely, suppose $M_{1}$ has the stated property. Let $H$ be a semisimple submodule of $M_{1}$. By hypothesis, there exist a direct summand $K$ of $M$ and a monomorphism on $H$ such that $M_{2} \subseteq K$, $\varphi(H) \cap K=0$ and $\varphi(H) \oplus K$ is an essential submodule of $M$. Now

$$
K=K \cap\left(M_{1} \oplus M_{2}\right)=\left(K \cap M_{1}\right) \oplus M_{2},
$$

so that $K \cap M_{1}$ is a direct summand of $M$, and hence also of $M_{1}$. Let $M_{1}=\left(K \cap M_{1}\right) \oplus X$ for some submodule $X$ of $M_{1}$ and let $\pi: M \rightarrow X$ be the canonical projection with kernel $K$. Define $f: H \rightarrow X$ by $f(h)=\pi(\varphi(h))$ where $h \in H$. It is easy to check that $f$ is a monomorphism. Let $0 \neq x \in X$. Then there exists an $r \in R$ such that $0 \neq x r \in \varphi(H) \oplus K$. It follows that $x r=\pi(x r)=f(h)+\pi(k)$ for some $h \in H, k \in K$. So

$$
0 \neq x r=f(h) \in f(H) .
$$

Hence, $f(H)$ is an essential submodule of $X$. Thus, $M_{1}$ satisfies the weak $C_{12}$ condition.

A question posed in [6, page 496] which asks whether the weak $C_{12}$ condition implies the $C_{12}$ condition or not? Our final concern is to answer this question negatively by providing several counter examples. First, note that the next two examples are based on the Abelian group, i.e., the $\mathbb{Z}$-module and the torsion-free module over a principal ideal domain. 
Example 3.7. The Specker group satisfies the weak $C_{12}$ but does not satisfy $C_{12}$.

Proof. Let $M$ be the Specker group $\prod_{i=1}^{\infty} \mathbb{Z}$. First note that $M_{\mathbb{Z}}$ does not satisfy $C_{12}$ by [12, Lemma 3.4]. Now $M_{\mathbb{Z}}$ is nonsingular by [7, Proposition 1.22]. Hence, [7, Corollary 1.26] gives that $M_{\mathbb{Z}}$ has zero socle. So $M_{\mathbb{Z}}$ has the weak $C_{12}$ condition.

Example 3.8. Let $R$ be a principal ideal domain. If $R$ is not a complete discrete valuation ring, then there exists an indecomposable torsion-free $R$ - module $M$ of rank 2 by [8, Theorem 19]. For $M$, Soc $M=0$. Hence, clearly $M$ satisfies the weak $C_{12}$ condition. However, $M_{R}$ has uniform dimension 2. By Lemma $2.1, M_{R}$ does not have the $C_{12}$ property.

Although Examples 3.7 and 3.8 show the existence of counter examples for the aforementioned question, surprisingly we can provide more algebraic topology type examples in the following result.

Theorem 3.9. Let $\mathbb{R}$ be the real field and $n$ any odd integer with $n \geq 3$. Let $S$ be the polynomial ring $\mathbb{R}\left[x_{1}, \ldots, x_{n}\right]$ indeterminates $x_{1}, \ldots, x_{n}$ over $\mathbb{R}$. Let $R$ be the ring $S / S s$, where $s=x_{1}^{2}+\cdots+x_{n}^{2}-1$. Then the free $R$-module $M=\oplus_{i=1}^{n} R$ contains a submodule $K_{R}$ which satisfies weak $C_{12}$ but does not satisfy $C_{12}$.

Proof. It is clear that $R$ is a commutative Noetherian domain. Let $\varphi: M \rightarrow R$ be the homomorphism defined by

$$
\varphi\left(a_{1}+S s, \ldots, a_{n}+S s\right)=a_{1} x_{1}+\cdots+a_{n} x_{n}+S s
$$

for all $a_{i}$ in $S, 1 \leq i \leq n$. Clearly, $\varphi$ is an epimorphism and hence its kernel $K$ is a direct summand of $M$, i.e., $M=K \oplus K^{\prime}$ for some submodule $K^{\prime} \cong R$. Obviously, $K$ is not uniform. Note that $K$ is the $R$-module of regular sections of the tangent bundle of the $(n-1)$ sphere $S^{n-1}$. Since the Euler characteristic $\chi\left(S^{n-1}\right) \neq 0$ it follows that the $(n-1)$-sphere cannot have a nonvanishing regular section of its tangent bundle (see, [4, Corollary VI.13.3]). Now $K_{R}$ has zero socle and hence it satisfies the weak $C_{12}$ condition. However, $K_{R}$ has uniform 
dimension $n-1$ which yields that $K_{R}$ does not satisfy the $C_{12}$ property by Lemma 2.1 .

\section{Remark 3.10.}

(i) If $n$ is 1 or 2 in Theorem 3.9, then $K_{R}$ is isomorphic to 0 or $R$, respectively. In these cases, $K_{R}$ has $C_{12}$ and so too does the weak $C_{12}$.

(ii) If $n$ is any even integer with $n \geq 4$, then the proof of Theorem 3.9 does not work. For example, $S^{3}, S^{5}$ and $S^{7}$ all have decomposable tangent bundles by the result of Adams (see [4]) and in these cases, $K_{R}$ is isomorphic to a (finite) direct sum of uniform modules. Hence, $K_{R}$ has $C_{12}$ by [13, Theorem 1.2] and also satisfies the weak $C_{12}$.

Acknowledgments. The authors are extremely grateful to the referee for his/her careful reading of the paper and useful suggestions.

\section{REFERENCES}

1. F.W. Anderson and K.R. Fuller, Rings and categories of modules, SpringerVerlag, New York, 1974.

2. G.F. Birkenmeier and A. Tercan, When some complements of a submodule is a summand, Comm. Algebra 35 (2007), 597-611.

3. G.F. Birkenmeier, A. Tercan and C.C. Yucel, The extending condition relative to sets of submodules, Comm. Algebra 42 (2014), 764-778.

4. G.E. Bredon, Topology and geometry, Springer Verlag, New York, 1993.

5. N.V. Dung, D.V. Huynh, P.F. Smith and R. Wisbauer, Extending modules, Pitman, London, 1994.

6. N. Er, Direct sums and summands of weak CS-modules and continuous modules, Rocky Mountain J. Math. 29 (1999), 491-503.

7. K.R. Goodearl, Ring theory: Nonsingular rings and modules, Dekker, New York, 1976.

8. I. Kaplansky, Infinite abelian groups, University of Michigan Press, Ann Arbor, 1969.

9. S.H. Mohamed and B.J. Müller, Continuous and discrete modules, Lond. Math. Soc. 147, 1990.

10. P.F. Smith, CS-modules and weak CS-modules, in Noncommutative ring theory, Springer Lect. Notes Math. 1448 (1990), 99-115.

11. P.F Smith and A. Tercan, Direct summands of modules which satisfy $C_{11}$, Alg. Colloq. 11 (2004), 231-237.

12. , Generalizations of CS-modules, Comm. Alg. 21 (1993), 1809-1847. 
13. F. Takil and A. Tercan, Modules whose submodules are essentially embedded in direct summands, Comm. Alg. 37 (2009), 460-469.

14. A. Tercan, On the endomorphism rings of modules with $C_{11}$ and $C_{2}$, Hacettepe Bull. Nat. Sci. Eng. 22 (1993), 1-7.

15. W Weak $\left(C_{11}\right)$ modules and algebraic topology type examples, Rocky Mountain J. Math. 34 (2004), 783-792.

16. H.Y. Yu, On modules for which the finite exchange property implies the countable exchange property, Comm. Alg. 22 (1994), 3887-3901.

17. Y. Zhou, Examples of rings and modules as trivial extensions, Comm. Alg. 27 (1999), 1997-2001.

18. W. Zimmermann and Z.B. Husgen, Classes of modules with exchange property, J. Algebra 88 (1984), 416-434.

Department of Mathematics, Hacettepe University, Beytepe Campus, AnkaRA 06532, TURKEY

Email address: yelizkara@hacettepe.edu.tr

Department of Mathematics, Hacettepe University, Beytepe Campus, AnkaRA 06532, TURKEY

Email address: tercan@hacettepe.edu.tr 